\title{
The comprehensive evaluation model of power supply capacity for regional-oriented distribution network
}

\author{
Xiaoming Zhou ${ }^{1 *}$, Xiaolan $\mathrm{Li}^{2}$, Fujia Liu ${ }^{3}$, Weichun $\mathrm{Ge}^{4}$, Xianzhi Ma ${ }^{1}$, Ye Tian ${ }^{5}$, Guangao $\mathrm{Li}^{1}$, Jiaxin Zhang ${ }^{1}$ Yue Qiu $^{6}$ \\ ${ }^{l}$ Operation monitoring center of State Grid Liaoning Electric Power Supply Co. Ltd, Shenyang, China \\ ${ }^{2}$ Operation monitoring center of State Grid Shenyang Electric Power Supply Company \\ ${ }^{3}$ State Grid Liaoning Electric Power Supply Co. Ltd, Shenyang, China \\ ${ }^{4}$ Technology and Information Department of State Grid Liaoning Electric Power Supply Co. Ltd, Shenyang, China \\ ${ }^{5}$ Electric Power Research Institute of State Grid Liaoning Electric Power Co., Ltd. Shenyang, China \\ ${ }^{6}$ Max Planck Institute for Dynamics of Complex Technical Systems, Sandtorstr.1, 39106 Magdeburg, Germany \\ *Corresponding Author’s Email:zhouxm@ln.sgcc.com.cn
}

\begin{abstract}
With the rapid increase of power loads, power supply capacity plays a significant role in power distribution network. However, attributing to the tremendous differences in regional economic development and diversified characteristics of power distribution network, power supply capacity should be evaluated based on regional differences to better promote the efficiency of distribution network. In this paper, the evaluated indexes including supply capacity reserve, supply capacity margin and supply capacity balance are defined and the evaluated models for single and inter-layer equipment are established. Furthermore, a case study with 10 different regions is employed to validate the proposed models. Results illustrate that considering the effects of "N-x" criterion, network structures and overload features, the proposed comprehensive models could quantitively evaluate the supply capacity and offer better supports for planning and operation of large-scale power distribution network.
\end{abstract}

Keywords: Evaluation model; Power supply capacity; "N-x" criterion; Evaluated indexes

\section{Introduction}

As a link between customers, power plants and transmission system, the reliability of the power distribution network directly affects the quality of the customers power supply. ${ }^{1-3}$ With the rapidly expanding of urbanization in China, the power loads are undergoing great increasing which therefore, result in a large mismatch between demands of markets and power supply capacities. In addition, the districts of power supply in China are vast and unbalanced that lead to some different profiles among power network supplies with vary economic development. Hence, it is unreasonable that the power supply networks are constructed using a unified standard due to above regional differences. Traditionally, the reliability rate of power persistence, voltage qualified rate and lines loss rate are employed to evaluate the performance of power distribution network. ${ }^{4,5}$ However, these evaluation indexes could not reflect the operation performance of power distribution network comprehensively. Therefore, how to quantitively evaluate the capability of power supply has become an important issue.

Many researches have been conducted to evaluate the performance of power supply capacity. ${ }^{6-8}$ Chang et al. ${ }^{9}$ established three evaluation indexes to assess the economic operation in the Longyangxia power station. They illustrated that the economic operation was unreasonable with $\mathrm{RC}>1, \mathrm{RU}<1$ and $\mathrm{RI}<0$. Georgilakis et al. ${ }^{10}$ optimized electrical distribution network operation and planning with the constrains of distributed generation. Mathematical models were presented to solve the optimal distributed generation placement problem. Gao et al. ${ }^{11}$ investigated the influence of accessed distributed generation on the power supply based on generalized sensitivity analysis. They validated the feasibility of the evaluation model via IEEE 14-node case study. Zhuo et al. ${ }^{12}$ proposed application principles of power supply network based on $500 / 110 \mathrm{kV}$ direct transformation. A comprehensive evaluation including accessibility index were presented on the power grid 
planning of a high-tech industrial park in Wuhan, China. Luo et al. ${ }^{13}$ proposed a straightforward method to evaluate the power supply capability of distribution system, which is based on "N-1" simulation analysis of interconnected main-transformers. Additionally, they obtained the maximum permissible load rate of each main-transformer with a comprehensive analysis. Liu et al. ${ }^{14}$ proposed a real-time evaluation method for power supply capacity of distribution network based on the uncertainty of distributed generation output and the cyclical change characteristics of power grid load. They employed a practical example to verify the feasibility and effectiveness of the proposed method and model.

In this paper, hierarchical equipment in power distribution network is identified for regional-oriented distribution network. The overall loads capacities are also analyzed quantitively using equipment and districts aggregation. We present the evaluation indexes including inner-layer power capacity, power capacity reserve, power capacity margin and power capacity balance which construct the evaluation models for single equipment, inner-layer and inter-layer equipment. In addition, the " $\mathrm{N}-\mathrm{x}$ " criterion, network structure, overloads characteristics and regional features are considered in the power capacity assessment which could support the construction planning and operation.

\section{Effects of power supply capacity of power distribution network}

Considering the effects of power supply capacity of distribution network, we expatiate on the power supply capacity of power distribution network with aspects of security, economy and regional characteristics in the follows.

\subsection{Safety operation}

The power supply of distribution network needs to meet the basic operational safety guidelines with some operation constraints including voltage, environment, maintenance requirements, etc. They will affect the standards of power supply capacity.

\subsection{Equipment overloads capability}

In actual production, partial equipment could afford instantaneously the maximum load capacity. It should be considered as in the calculation process for the power supply capacity.

\subsection{Structure of power distribution network}

The power distribution network should meet the safety reliability and operational flexibility when it is designed. The rationality of grid structure could influence the power supply capacity.

\subsection{Regional characteristics}

There are significant differences among economic development and distribution characteristics of power grid in different regions. Therefore, these differences are important and need to be well analyzed.

\section{Evaluation of power supply capacity of distribution network system}

\subsection{The hierarchy of power supply capacity evaluation}

The supply capacity models of power distribution network could be divided into three hierarchies, power supply capacity for single equipment, operational efficiency for inner-layer equipment and the power supply capacity of distribution system. At each hierarchy, the maximum power supply capacity, the power supply capacity reserve and the power supply ability margin are established to reflect the comprehensive quality of power supply capacity. On the basis of the operation efficiency evaluation model for single equipment, we have established the power supply capacity evaluation model for inner-layer equipment and power supply capacity 
evaluation model for distribution network system. Therefore, the comprehensive power supply capacity evaluation indexes are constructed as shown in Table 1.

Table 1 Evaluation model of power distribution network

\begin{tabular}{|c|c|c|}
\hline Models & Objects & Indexes \\
\hline \multirow{5}{*}{ Power distribution capacity } & \multirow{5}{*}{$\begin{array}{l}\text { Single equipment } \\
\text { (Transformer/Line) }\end{array}$} & Max supply capacity (SC) \\
\hline & & Supply capacity reserve \\
\hline & & $(\mathrm{SCR})$ \\
\hline & & Supply capacity margin \\
\hline & & (SCM) \\
\hline \multirow{7}{*}{ Inner-layer supply capacity } & \multirow{7}{*}{$\begin{array}{l}\text { The hierarchy of power } \\
\text { network (High/Main/Medium } \\
\text { voltage lines/Transformer) }\end{array}$} & Inner-layer Max supply \\
\hline & & capacity (GSC) \\
\hline & & $\begin{array}{l}\text { Inner-layer Supply capacity } \\
\text { reserve (GSCR) }\end{array}$ \\
\hline & & Inner-layer Supply capacity \\
\hline & & $\operatorname{margin}(\mathrm{GSCM})$ \\
\hline & & Inner-layer supply margin \\
\hline & & balance (BSCM) \\
\hline \multirow{4}{*}{$\begin{array}{l}\text { Coordination of supply } \\
\text { capacity }\end{array}$} & The inter-hierarchy of & \\
\hline & power network & System-level supply margin \\
\hline & (High/Main/Medium voltage & balance (CGSCM) \\
\hline & lines/Transformer) & \\
\hline
\end{tabular}

\subsection{The evaluation model of power supply capacity for single equipment}

The power supply capacity of distribution network is defined as the ability to afford the power loads with safety operation. The evaluation model of power supply capacity includes three basic indexes: maximum supply capacity, supply capacity reserve and supply capacity margin. There are two types of single equipment including transformers and electric lines. According to the different voltage grades, it is divided into four categories: main transformers, distribution transformers, high-voltage lines and medium-voltage lines.

\subsubsection{The maximum supply capacity for single equipment}

Generally, it is considered that the maximum power supply capacity $\left(P_{1.0}\right)$ is identical with the maximum transport loads of equipment which could be calculated according to the rated capacity. However, in the actual production, the equipment meets the basic safety criterion (such as " $\mathrm{N}-\mathrm{x}$ " criterion,), and considers the maximum transport loads of other constraints (such as voltage constraint, operating environment, maintenance and maintenance). The maximum power supply capacity is regarded as $P_{U}$. In addition, partial equipment could be conducted overloaded in a short time. Therefore, considering the overloads ratios and operation requirements, the max supply capacity could be precisely calculated. The maximum power supply capacity of the equipment is defined as:

$$
S C=k \cdot P_{U}
$$


where SC (Supply Capacity) is the maximum power supply capacity of single equipment. $P_{U}$ is the maximum loads with the constrains of basic safety criteria such as " $\mathrm{N}-\mathrm{x}$ " safety regulations. $k$ is the overload ratio which is set to be $1 \sim 1.3$.

(1) To satisfy the "N-x" safety criterion, the maximum power supply capacity of the substation should be calculated initially. When the number of substation transformer is set as 1 , the maximum power supply capacity of substation is the maximum power supply capacity of the main transformer under the substation which is defined as:

$$
S C_{s}=k \cdot P_{1.0}
$$

where $S C_{s}$ is the maximum power supply capacity of substation. $P_{1.0}$ is the maximum power variation capacity of the main transformer.

When the number of main transformer of the substation is not less than 2, the maximum power supply capacity of the substation is equivalent to the power transmission capacity of substation not including the maximum conversion capacity. The calculation formula of the maximum power supply capacity of each substation could be defined as:

$$
S C_{s}=k \cdot\left(\sum_{t=1}^{T} P_{1.0_{-} t}-\max _{t=1}^{T} P_{1.0_{-} t}\right)
$$

where $P_{1.0 t}$ is the maximum power conversion capacity of the type $t$ main transformer. $T$ is the number of the main transformer in a substation. $\max _{t=1}^{T} P_{1.0_{-} t}$ is the maximum supply capacity of single main transformer in a substation.

When the number of main transformer under the substation is not less than 2, the maximum power supply capacity of a main transformer in the substation is equivalent to the power transmission capacity of all the transformers in the substation. Therefore, the maximum transferable load of main transformer could be deduced which is less than that of the single transformer.

(2) Considering the maximum power supply capacity of high-voltage lines and medium-voltage lines, due to the power distribution network needs to meet the requirements of safety criterion and economic efficiency, the values of $P_{U} / P_{1.0}$ could be employed to classify different equipment. For equipment that do not be required to meet the ' $\mathrm{N}-\mathrm{x}$ ' security criterion (e.g. single radiation electric lines), we can derive that $P_{U} / P_{1.0}=1$. The values of high-voltage lines are defined as, 1.0 for single radiation, 0.5 for double radiations, double chains, single ring net and single chain network, 0.5 for other companies, 1.0 for other customers. Finally, the medium-voltage lines are defined as, 1.0 for single radiation, single shot, 0.67 for two contacts, 0.75 for third contacts and above. 0.5 for single ring, single contact, double rings, double shots, 0.5 for other companies and 1.0 for other customers.

(3) The maximum power supply capacity of distribution transformer

The maximum power supply capacity of distribution transformer is the maximum power capacity of equipment. The overloads ratio and safety operation criterion are not considered.

\subsubsection{Power supply capacity reserve of single equipment}


Power supply capacity reserve reflects the affordable electric loads at the current loads level. The power supply capacity reserve of single equipment is evaluated based on the maximum power supply capacity and maximum electric loads of equipment. Since the maximum loads of the substation are the sum of the loads all of the main transformers synchronously, therefore, the power supply capacity reserve of single equipment could be defined as,

$$
S C R=S C-P_{\max }
$$

where $S C R$ is the power supply capacity reserve of the equipment. $P_{\max }$ refers to the maximum loads of the equipment.

\subsubsection{Power supply capacity margin of single equipment}

The power supply capacity margin is defined as the ratio of affordable electric loads to total loads as follows,

$$
S C M=S C R / S C
$$

where $S C M$ is the power supply capacity margin of equipment. The power supply capacity margin of main transformer is equal to the power supply capacity margin of substation.

\subsection{Power supply capacity evaluation of inner-layer equipment}

\subsubsection{The evaluation model of power supply capacity for the inner-layer equipment}

On the basis of the evaluation model of the power supply capacity for single equipment, we divide the distribution network equipment into four categories according to different attributes: high-voltage lines, main transformer, medium-voltage lines and distribution transformer. The general power supply capacity evaluation model for kinds of equipment could determine the power supply capacity of the inner-layer equipment. The supply capacity balance model of equipment is established to describe the operation condition of the inner-layer equipment.

In order to determine the power supply capacity of one kind of equipment, the maximum calculation method for inner-layer supply capacity based on the power supply capacity of single equipment is proposed which is defined as follows,

$$
G S C_{i}=\sum_{j=1}^{M_{i}} S C_{\mathbf{j}}
$$

where $G S C_{i}$ is the general maximum power supply capacity of the type ith layer equipment. $i=1,2,3,4$ represent high-voltage line, the substation, medium-voltage line and distribution transformer, respectively. $M_{i}$ is the total number of type ith equipment. $S C_{j}$ is the maximum power supply capacity of the type jth equipment. Power supply capacity reserve of the inter-layer equipment is defined as,

$$
G S C R_{i}=\sum_{j=1}^{M_{i}} S C R_{\mathbf{j}}
$$


where $G S C R$ is the power supply capacity reserve for the type ith layer equipment. $i=1,2,3,4$ represent high-voltage line, the substation, medium-voltage line and distribution transformer, respectively. $M_{i}$ is the total number of type ith equipment. $S C R_{j}$ is the power supply capacity of the type $j t h$ equipment. The general supply capacity margin for the inner-layer equipment is defined as,

$$
G S C M_{i}=G S C R_{i} / G S C_{i}
$$

where $\operatorname{GSCM}_{i}$ is the general supply capacity margin of the type $i t h$ layer equipment. $i=1,2,3,4$ represent high-voltage line, the substation, medium-voltage line and distribution transformer, respectively. $M_{i}$ is the total number of type ith equipment. $S C R_{j}$ is the power supply capacity of the type $j$ th equipment

\subsubsection{Power supply capacity balance model of inner-layer for the distribution network system}

Power supply capacity balance model is employed to calculate the relative balance ratio of the power supply capacity among the inter-layer equipment. Based on the standard deviation and arithmetic mean of the power supply capacity of the inner-layer equipment, the power supply capacity balance ratio is obtained from the exponential function of the natural constant. The balance ratio could determine the difference of power supply capacity for the inter-layer equipment.

Before calculating the power supply capacity balance of the inter-layer equipment, the indexes of power supply capacity margin for each equipment need to be normalized. The specific methods are as follows,

$$
S C M_{i j}^{\prime}=\frac{S C M_{i j}-a}{b-a}
$$

where $a$ and $b$ are determined by the equipment type, $k \cdot P_{u} / P_{1.0}$ and $P_{\max } / P_{1.0}$. The values are obtained from Table.2 where

$$
\begin{gathered}
a=\frac{k \cdot P_{u} / P_{1.0}-P_{\max } / P_{1.0}}{k \cdot P_{u} / P_{1.0}} \\
B_{S C M_{i}}=e^{\wedge}\left(-\frac{\sqrt{\frac{\sum_{j=1}^{M_{i}}\left(\mathbf{S C M}{ }_{i j}^{\prime}-\overline{S C M_{i}}\right)^{\prime}}{M_{i}}}}{{\overline{S C M_{i}}}^{\prime}}\right)
\end{gathered}
$$

Table 2 The values of Power supply capacity balance model

\begin{tabular}{lccccc}
\hline $\begin{array}{l}\text { Equipment } \\
\text { types }\end{array}$ & $k \cdot P_{u} /$ & $P_{\max } / I$ & $\begin{array}{r}\text { Lower } \\
\text { bound }(a)\end{array}$ & $\begin{array}{r}\text { Upper } \\
\text { bound }(b)\end{array}$ & $\begin{array}{c}\text { Internal } \\
\text { length }(c)\end{array}$ \\
\hline High-voltage & 1.00 & 1.00 & 0.00 & 1.00 & 1.00 \\
\hline
\end{tabular}




\begin{tabular}{cccccc}
\hline line & 0.50 & 1.00 & -1.00 & 1.00 & 2.00 \\
\hline & 1.30 & 1.50 & -0.15 & 1.00 & 1.15 \\
\cline { 2 - 5 } Substation & 0.65 & 1.50 & -1.31 & 1.00 & 2.31 \\
\cline { 2 - 5 } & 0.87 & 1.50 & -0.73 & 1.00 & 1.73 \\
\cline { 2 - 5 } & 0.98 & 1.50 & -0.54 & 1.00 & 1.54 \\
\cline { 2 - 5 } $\begin{array}{c}\text { Medium-volta } \\
\text { ge line }\end{array}$ & 1.00 & 1.00 & 0.00 & 1.00 & 1.00 \\
\cline { 2 - 5 } & 0.75 & 1.00 & -0.33 & 1.00 & 1.33 \\
\cline { 2 - 5 } & 0.67 & 1.00 & -0.50 & 1.00 & 2.00 \\
\hline \multirow{2}{*}{$\begin{array}{c}\text { Distribution } \\
\text { transformer }\end{array}$} & 0.50 & 1.00 & -1.00 & 1.00 & 1.50 \\
\hline
\end{tabular}

where $B_{S C M}$ is the balance of supply capacity margin for inner-layer equipment. $i=1,2,3,4$ represent high-voltage line, the substation, medium-voltage line and distribution transformer, respectively.

\subsection{Power supply capacity evaluation of power distribution system}

\subsubsection{Power supply capacity evaluation model of power distribution system}

In order to determine the supply capacity of the distribution network system, we propose a calculation method that the whole distribution network is considered as an entirety. As the incoming and outgoing lines are just considered, the maximum power supply capacity of the system is the difference value between the sum of the maximum power supply capacity for all the incoming lines and the sum of the maximum power supply capacity for all the outgoing lines. The calculation formula of the systemic maximum power supply capacity is as follows,

$$
S S C=\sum_{k=1}^{I L} S C_{\mathbf{I L}_{-} \mathbf{k}}-\sum_{l=1}^{O L} S C_{\mathbf{O L}_{-} \mathbf{I}}
$$

where $S S C$ is the maximum power supply capacity of the power distribution system. $S C_{\mathbf{I L} \mathbf{k}}$ is the maximum power supply capacity of the type $k t h$ incoming lines. $S C_{\mathbf{O L}_{-} \mathbf{I}}$ is the maximum power supply capacity of the $1 s t$ outgoing line. $I L$ is the number of incoming lines for power the distribution network system. $O L$ is the number of outgoing lines for the distribution network system.

The power supply capacity reserve of the system is calculated by the formula,

$$
S S C R=\sum_{k=1}^{I L}\left(S C_{\mathbf{I L} \_\mathbf{k}}-P_{\text {max IL_k }}\right)-\sum_{l=1}^{O L}\left(S C_{\mathbf{O L} \_\mathbf{I}}-P_{\text {maxOL_k }}\right)
$$


where $S S C R$ is the power supply capacity reserve of the distribution network system. $P_{\mathbf{m a x} I L_{-} \mathbf{k}}$ is the maximum annual loads for the type $k t h$ incoming lines of the system. $P_{\text {maxOL_k }}$ is the maximum annual load for the 1 st outgoing lines of the system.

\subsubsection{Power supply capacity coordination model of power distribution system}

Using the establishment of power supply capacity coordination model of power distribution system, the coordination ratio of the power supply capacity inter-layer equipment could be determined including high-voltage lines, substation, medium-voltage lines and distribution transformers. The normalization of the power supply capacity margin of inter-layer equipment is defined as,

$$
G S C M_{i}^{\prime}=\frac{G S C M_{i}-a}{b-a}
$$

where $G S C M_{i}$ is the power supply capacity margin of the equipment in the ith level. $i=1,2,3,4$ represent high-voltage line, the substation, medium-voltage line and distribution transformer, respectively. In this case, $a$ is chosen as -1.31 and $b$ is 1 .

$$
C_{G S C M}=e^{\wedge}\left(-\frac{\sqrt{\frac{\sum_{i=1}^{N}\left(G S C M_{\mathrm{i}}^{\prime}-\overline{G S C M}^{\prime}\right)^{2}}{N}}}{\overline{G S C M}^{\prime}}\right)
$$

where $C_{G S C M}$ is the power supply capacity coordination of each layer.

$$
\overline{G S C M}^{\prime}=\frac{\sum_{i=1}^{N} G S C M_{i}^{\prime}}{N}
$$

Based on the ratio of the standard deviation to the average amount, we obtain the power supply capacity coordination ratio by the exponential function of the natural constants. It can determine the difference of power supply capacity among inter-layer equipment. The coordination ratio is higher, the loads planning of inter-layer equipment is more reasonable. The coordination ratio is lower, the power supply capacity of inter-layer equipment is much different which leads to a more overexploitation or overinvestment for one-layer equipment.

\section{Case study}

In this case, the distribution network of 10 regions is chosen to validate the model. The equipment types include 10 substations, 20 high-voltage lines, 50 main transformers, 500 medium-voltage lines and 10000 distribution transformers.

\subsection{Power supply capacity evaluation of single equipment}

According to the data of minute-class operation from a power supply company, four medium-voltage lines are chosen for analyzing of power supply capacity of single equipment. All of the lines are single-radiation structures. The timing loads of all day for 4 electric lines are illustrated in Fig. 1. 


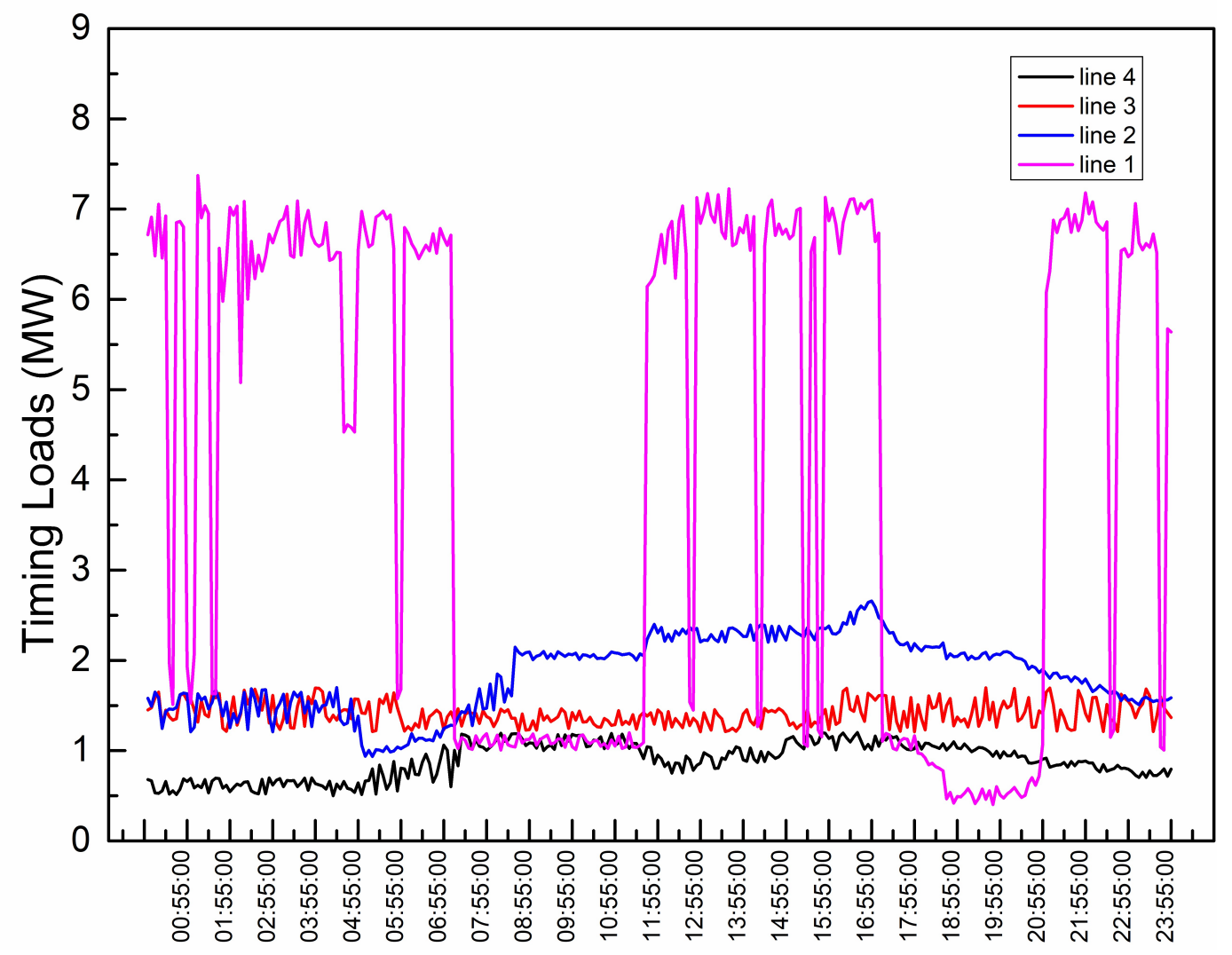

Fig. 1 The timing loads of all day for 4 electric lines

According to the voltage level and maximum affordable current, we can obtain the rated capacity of the lines. The results of basic indexes could be calculated using Eq. (1), (4) and (5) which is illustrated in table 3.

Table 3 The evaluation indexes of power distribution network

\begin{tabular}{ccccc}
\hline & \multicolumn{4}{c}{ Evaluation Results } \\
\cline { 2 - 4 } Lines & SCM & SCR & $\mathrm{P}_{\max }$ & SC \\
\cline { 2 - 4 } Line1 & 0.00010 & 0.00100 & 7.36000 & 7.36100 \\
Line2 & 0.43670 & 2.04583 & 2.63867 & 4.68450 \\
Line3 & 0.64420 & 3.52071 & 1.94454 & 5.46520 \\
Line4 & 0.82870 & 6.10060 & 1.26040 & 7.36100 \\
\hline
\end{tabular}

Compared with lines 1 and 4, the maximum power supply capacity (SC) is 7.361(MW). The operating loads of line 1 are close to its maximum power supply capacity in some periods, resulting in insufficient power supply. In actual production, the power supply capacity of the equipment can be evaluated effectively by setting the threshold in the reasonable range of the power supply capability margin (SCM).

\subsection{The power supply capacity and supply capacity balance evaluation of inter-layer equipment}

The results of power supply capacity margin evaluation for the inter-layer equipment are shown in table 4. 
Table 4. The evaluations of general power supply capacity

\begin{tabular}{ccccc}
\hline Region & \multicolumn{3}{c}{ Evaluation Results } & \\
\cline { 2 - 4 } & substation & $\begin{array}{c}\text { high-volt } \\
\text { age line }\end{array}$ & $\begin{array}{c}\text { distribution } \\
\text { transformer }\end{array}$ & $\begin{array}{c}\text { medium-volta } \\
\text { ge line }\end{array}$ \\
\hline Region 1 & 0.39454 & 0.23177 & 0.73608 & 0.45415 \\
Region 2 & 0.53949 & 0.46100 & 0.62136 & 0.53322 \\
Region 3 & 0.73116 & 0.37673 & 0.69665 & 0.67513 \\
Region 4 & 0.71451 & 0.85903 & 0.70690 & 0.68095 \\
Region 5 & 0.55287 & 0.13892 & 0.66742 & 0.57702 \\
Region 6 & 0.48352 & 0.43734 & 0.67042 & 0.57983 \\
Region 7 & 0.71970 & 0.64009 & 0.63823 & 0.71995 \\
Region 8 & 0.58809 & 0.64122 & 0.68087 & 0.59735 \\
Region 9 & 0.64223 & 0.69564 & 0.66906 & 0.62663 \\
Region 10 & 0.53064 & 0.49348 & 0.65086 & 0.56287 \\
\hline
\end{tabular}

According to the evaluation results in table 3, the power supply capacity of high-voltage lines in 10 regions are largely different with each other. Among them, the highest power supply capacity margin of the high-voltage line is the region $4(0.86)$, and the lowest of them is the region $5(0.14)$. The highest power supply capacity margin of the substation is the region $3(0.73)$, and the lowest of them is the region1 (0.39). The highest power supply capacity margin of the medium-voltage line is the region $7(0.72)$, and the lowest of them is the region1 (0.45). The highest power supply capacity margin of the distribution transformer is the region $1(0.74)$, and the lowest of them is the region2 (0.62). The reason for the low margin of power supply capacity of region 5 is concluded as the large population and high concentration of electric loads. To ensure the voltages quality in the terminal district, the electric lines selections are based on the affordable voltage dropdown. The operation loads are higher than the lower bound of economy operation zones which lead to heavy overloads of equipment, and the supply capacity margin of equipment is therefore decreased. We calculate weight functions of equipment assets value in 10 regions and obtain power supply capacity margin for each regional system by Eq. (5). In addition, systemic power supply capacity balance is calculated by Eq. (6) and illustrated in Fig. 2. 


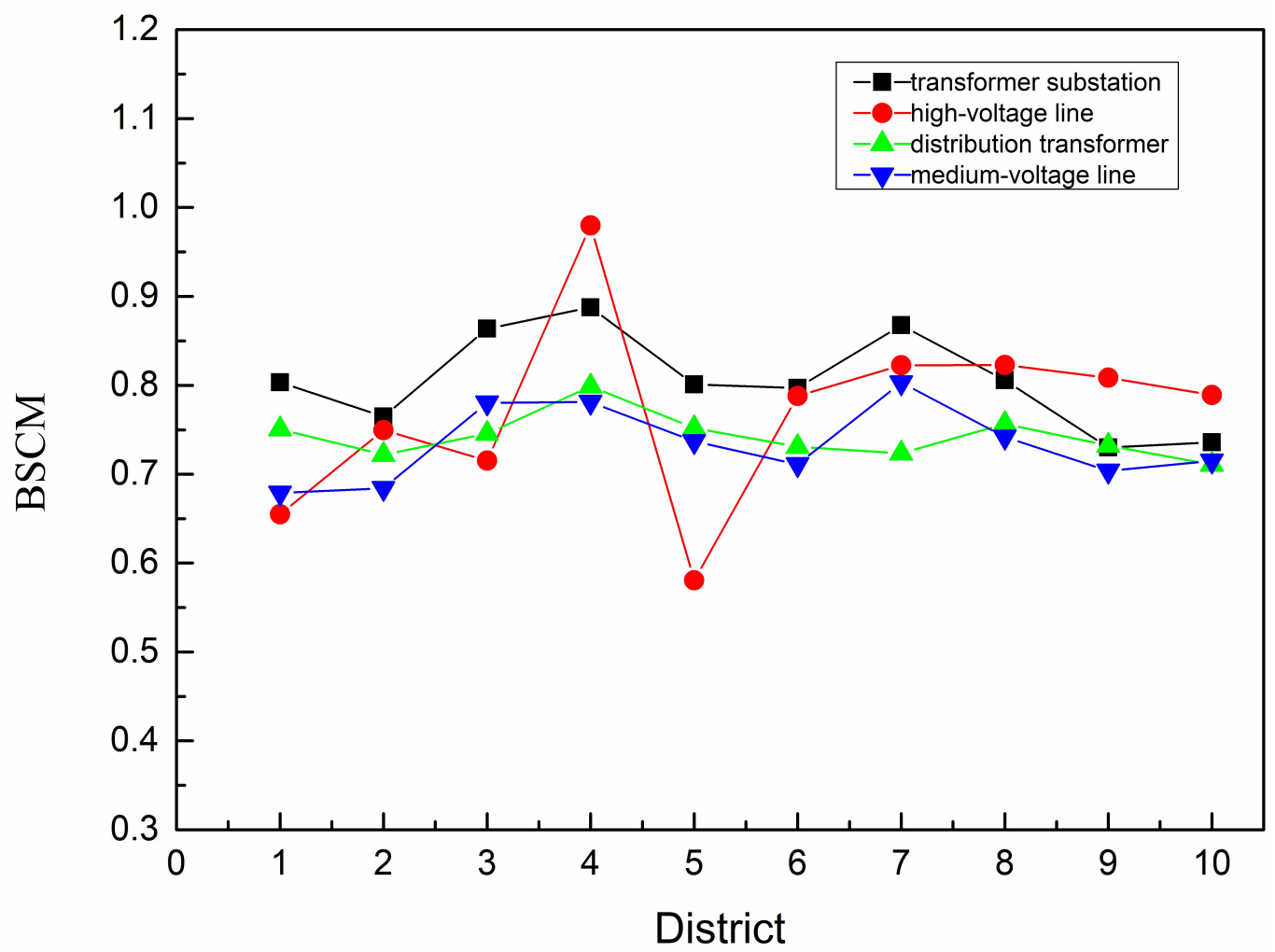

Fig. 2 The evaluation of supply capacity margin balance

It is illustrated in Fig.2 that the changes of high-voltage lines in area 4 and 5 are large. Furthermore, as shown in Fig.3, the main reason for the higher balance of power supply capacity in the region 5 is that the power supply capacity of the equipment in subordinate area is higher. The main reason for the lower balance of power supply capacity in the region 5 is that the power supply capacity of the equipment in subordinate area is lower. 


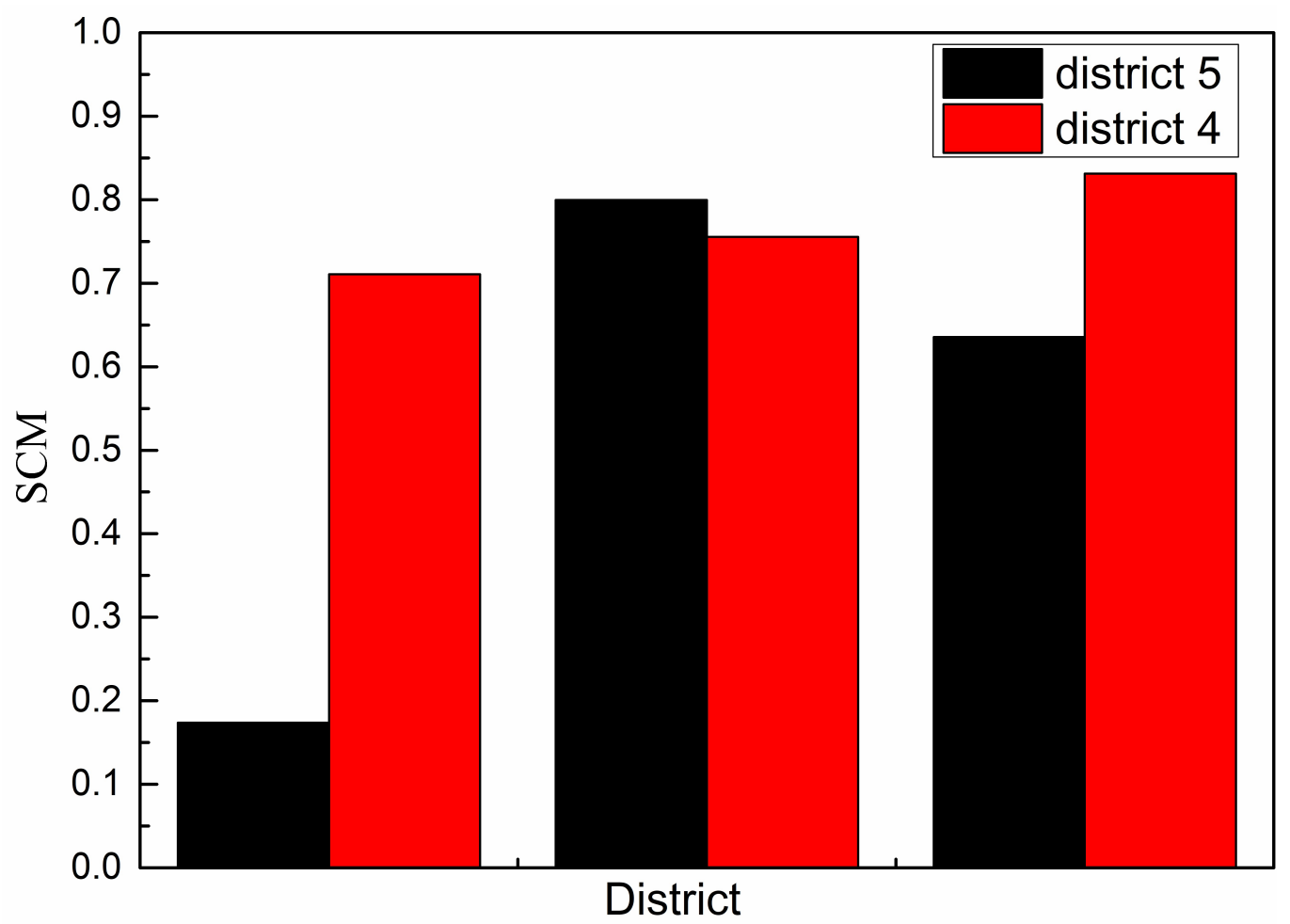

Fig. 3 The supply capacity margin

\subsection{The evaluation of systemic power supply capacity coordination ratio}

Based on the data of the inter-layer equipment in table 3, the weight functions of equipment assets value in 10 regions are therefore calculated. The results of power supply capacity for each regional system are calculated by Eq.(8). We obtain the coordination degree of power supply capacity of the system with Eq. (13), (14) and (15) and illustrate it in Fig. 4. 


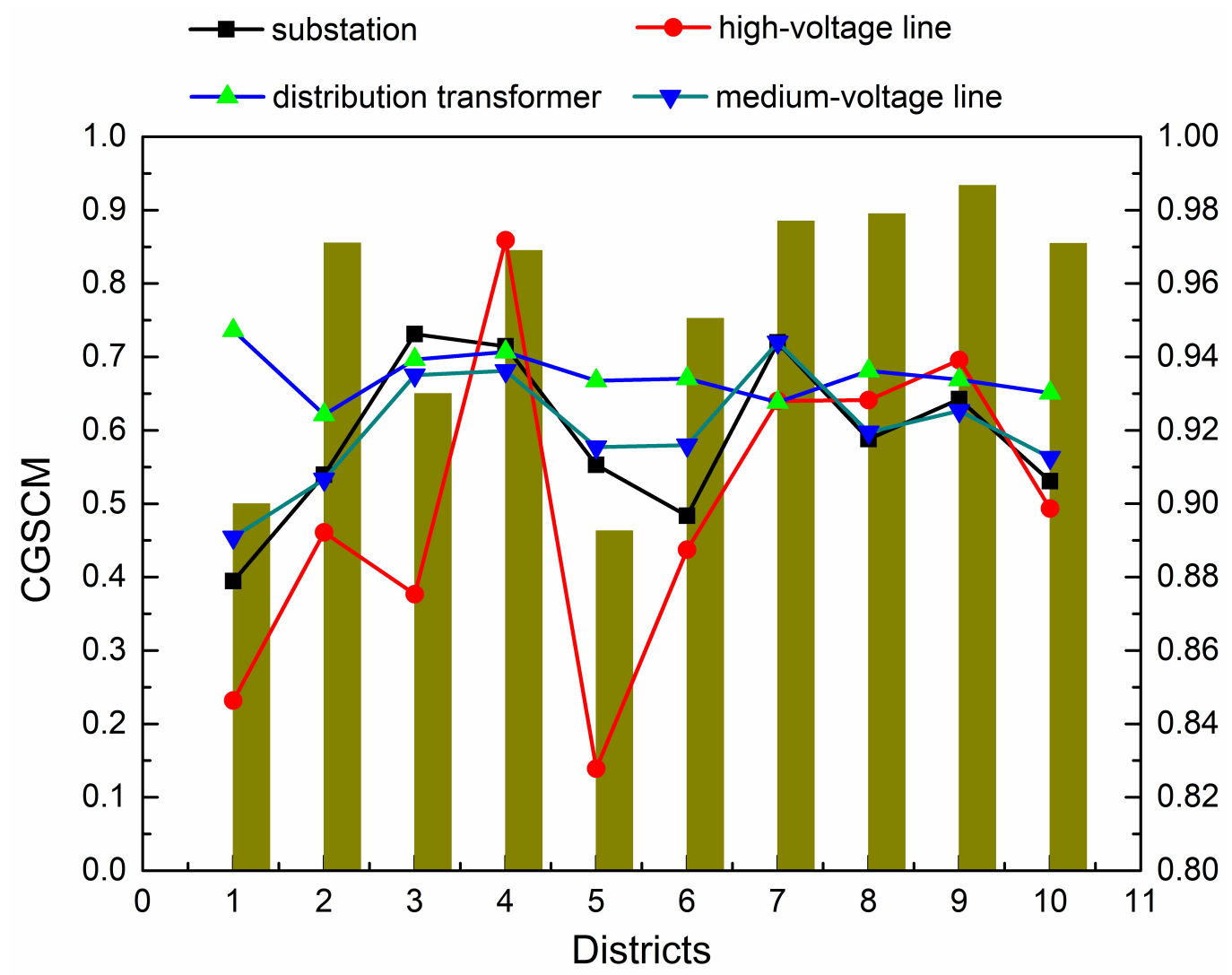

Fig. 4 The systemic supply capacity balance ratio

In Fig.4, it illustrates that the power supply capacity coordination ratio in region 9 is the highest (0.9867), the system power supply margin coordination ratio in region 5 is the lowest $(0.8926)$. By comparing the different levels of varying regions, it is clear that the range of power supply in the region 10 is similar to one identical value. The power supply capacity of the high-voltage lines in region 5 is significantly higher than other three kinds of equipment, so the system coordination ratio is decreased.

\section{Conclusion}

According to some problems existing in the evaluation of power supply capability of distribution network, power supply capability evaluation indexes are proposed based on the supply capacity reserve, supply capacity margin and supply capacity balance ratio. Additionally, the evaluated models for single and inter-layer equipment are established to satisfy the rapid increase of power loads. The actual distribution network of 10 regions are presented and analyzed to validate the accuracy of the proposed models. With the effects of "N-x" criterion, network structures and overload features, the proposed comprehensive models could quantitively evaluate the supply capacity and offer better supports for planning and operation of large-scale power distribution network.

\section{Acknowledgments}

This research is partially supported by State Grid Science and Technology Project 5222SY17004A, Improving Technological Innovation Ability and Mass Innovation Research Projects 2018ZX-17. 


\section{References:}

1. Ruiz-Romero S, Colmenar-Santos A, Mur-Pérez F, López-Rey Á. Integration of distributed generation in the power distribution network: The need for smart grid control systems, communication and equipment for a smart city -Use cases. Renewable and Sustainable Energy Reviews. 2014;38:223-234

2. Miu KN, Chiang HD. Electric distribution system load capability: Problem formulation, solution algorithm, and numerical results. Ieee T Power Deliver. 2000;15(1):436-442

3. Shen X, Shahidehpour M, Han Y, Zhu S, Zheng J. Expansion Planning of Active Distribution Networks With Centralized and Distributed Energy Storage Systems. Ieee T Sustain Energ. 2017;8(1):126-134

4. Jin X, Mu Y, Jia H et al. An Active Reconfiguration Strategy for Distribution Network Based on Maximum Power Supply Capability. Transactions of China Electrotechnical Society. 2014;29:137-147

5. Zhou Z, Ishida M, Maeda T. Capacity Planning and Practicality Evaluation of Grid-Independent Power System Based on Supply Reliability. Electr Eng Jpn. 2012;181(2):1-9

6. Gao Y, Liu J, Yang J, Liang H, Zhang J. Multi-Objective Planning of Multi-Type Distributed Generation Considering Timing Characteristics and Environmental Benefits. Energies. 2014;7(10):6242-6257

7. Jin P, Li Y, Li G, Chen Z, Zhai X. Optimized hierarchical power oscillations control for distributed generation under unbalanced conditions. Appl Energ. 2017;194:343-352

8. Liao H, Liu D, Huang Y, Zhang Y. Load transfer capability analysis considering interconnection of distributed generation and energy storage system. Int T Electr Energy. 2014;24(2):166-177

9. Chang J, Li Y, Yuan M, Wang Y. Efficiency evaluation of hydropower station operation: A case study of Longyangxia station in the Yellow River, China. Energy. 2017;135:23-31

10. Georgilakis PS, Hatziargyriou ND. Optimal Distributed Generation Placement in Power Distribution Networks: Models, Methods, and Future Research. Ieee T Power Syst. 2013;28(3):3420-3428

11. Gao Y, Yang W, Zhu J, Ren J, Li P. Evaluating the Effect of Distributed Generation on Power Supply Capacity in Active Distribution System Based on Sensitivity Analysis. Energies. 2017;10(10):1473

12. Zhuo Y, Xu J, Wei F et al. Design of power supply network based on 500/110 kv for load center and comprehensive accessibility evaluation. CSEE Journal of Power and Energy Systems. 2016;2(1):30-39

13. Fengzhang L, Chengshan W, Jun X et al. An evaluation method for power supply capability of urban electric power distribution system based on "N-1" simulation analysis of transformers. 1st International Conference on Sustainable Power Generation and Supply (SUPERGEN). 2009:7.

14. Liu J, Gao Y, Wu Y, Li J, Sun J. Real-time Evaluation for Power Supply Capacity of Distribution Network With Distributed Generation. 2014 INTERNATIONAL CONFERENCE ON POWER SYSTEM TECHNOLOGY (POWERCON). 2014:1485-1490. 